\title{
Use of hydrogel spacer for improved rectal dose-sparing in patients undergoing radical radiotherapy for localized prostate cancer: First Canadian experience
}

Alejandro Berlin, Anne Di Tomasso, Heather Ballantyne, Susan Patterson, Tony Lam, Aravind Sundaramurthy, Joelle Helou, Andrew Bayley, Peter Chung

Radiation Medicine Program, Princess Margaret Cancer Centre, Department of Radiation Oncology, University of Toronto, Toronto, ON, Canada

Acknowledgements: We thank Sylvia Blair and Kyle DeRouen (Augmentix Inc. Canada and USA, respectively) for their logistic and technical support.

Cite as: Can Urol Assoc J 2017; Epub ahead of print. http://dx.doi.org/10.5489/cuaj.4681

Published online November 1, 2017

$* * *$

\begin{abstract}
We describe the initial experience using a hydrogel spacer (SpaceOAR) to separate the prostaterectum interspace in patients planned to undergo radical hypofractionated image-guided intensity-modulated radiotherapy (IG-IMRT). We depict and discuss the impact of SpaceOAR in the context of hypofractionated IG-IMRT, and the particular considerations for its applications in the Canadian setting.
\end{abstract}




\section{Introduction}

Prostate cancer (PCa) is the most common non-cutaneous malignancy in Canadian men. ${ }^{1}$ External beam radiotherapy (EBRT) is a mainstay of treatment across the localized disease spectrum. Dose-escalation has lead to improved outcomes in prostate cancer but has been associated with increased risk of gastrointestinal, and in particular, rectal toxicity. ${ }^{2}$ Recent advances in radiation technology and delivery, including image-guided intensity modulated radiation therapy (IG-IMRT) have improved the therapeutic index, ${ }^{3}$ however in a proportion of cases it remains difficult to adequately spare the surrounding organs-at-risk (OAR). Moreover, in patients where dose constraints for OAR can be achieved, they still may be vulnerable to such toxicities, and this appears to be related to the volume of normal tissue encompassed within the high dose region.

In recent years, there has been a growing interest in placing biomaterials in the prostaterectum interface, creating a separation between the target (prostate) and the OAR (rectum), that can potentially reduce rectal doses. Of these, polyethylene glycol (PEG) hydrogels have been the most widely studied. ${ }^{4}$ Once within the perirectal space, cross-linked PEG rapidly polymerizes into a soft hydrogel, which after 3 months begins to hydrolyze until complete reabsorption in approximately 6-8 months. SpaceOAR (Augmenix Inc., USA) is an FDA and Health Canada approved PEG hydrogel rectal spacer, that demonstrated $>98 \%$ successful placement rate, with none or only mild symptoms (i.e. tenderness, fullness) at the site of injection resolving within 24 hours after the procedure. Importantly, the resulting space rendered better radiotherapy plan dosimetry, which improved late rectal toxicity and bowel-domain health-related QoL in a randomized study comprising more than two hundred men.,

Herein, we report our initial experience using SpaceOAR, the impact on IG-IMRT plan dosimetry, and discuss potential uses in the Canadian healthcare system setting.

\section{Methods and results}

Five patients with localized prostate cancer, planned for radical radiotherapy underwent rectal spacer insertion after providing informed consent. In brief, the patient was placed in the lithotomy position, and perineal skin prepped in a sterile manner. The skin and deep tissues up to the prostatic apex bilaterally were infiltrated with 20cc of 1\% lidocaine under TRUS guidance. Three gold fiducials were inserted transperineally at the prostate base, mid-gland and apex by TRUS guidance. Subsequently, the SpaceOAR injection needle (18G, 15cm length) was inserted parallel to the TRUS probe affixed to the brachytherapy stepper. The tip of the needle was positioned at the mid-gland level between Denonvilliers' fascia and the anterior rectal wall. Hydro-dissection was performed with 5-15cc of saline to confirm correct positioning. Under direct visualization, the assembled SpaceOAR kit was attached, and while maintaining the needle position, 10 cc of hydrogel was injected in one continuous motion over 8-10 seconds. The total procedure time ranged between 15 and 25 minutes. All patients tolerated the procedure well, with only mild discomfort related to the TRUS probe and initial lidocaine injection. 
Patients underwent computed tomography (CT) and magnetic resonance (MR) simulation for EBRT planning, 3-10 days after SpaceOAR insertion. CT was acquired, without intravenous contrast, at 2mm slice thickness per institutional practice. MR (3 Tesla) without endorectal coil consisted of axial T2w and DWI sequences for prostate and tumour delineation, and 3D-CISS sequence for optimal fiducial identification and CT-MR registration (see figure 1). The resultant distance between the anterior rectal wall and prostate measured in T2w MR sequence at the base, mid-gland and apex ranged between 10-13, 9-11 and 8-14 mm, respectively. Radiotherapy planning was performed in RayStation (RaySearch Laboratories, Sweden) following departmental (i.e. PROFIT) dose goals and constraints. All plans met criteria for approval. The resultant dose-volume histograms (DVH) of the five patients were averaged and compared to five randomly selected historical cases treated with the same IG-IMRT schedule (60Gy in 20Fx) for illustrating the differences in rectal doses (see figure 2).

\section{Discussion}

This report represents the first experience using SpaceOAR in Canada. Insertion as a short outpatient procedure under local anesthesia, translated in excellent results and patient tolerance. The dosimetric results of hypofractionated IG-IMRT compared favourably to historical plans. Our experience and planned future use of a rectal spacer for IG-IMRT have unique considerations in our setting.

The recently published Canadian PROFIT study, demonstrated 60Gy in 20 fractions to be non-inferior to conventionally fractionated dose-escalation (78Gy in 39 fractions). ${ }^{7}$ Based on this and other studies, ${ }^{8-9}$ mild hypofractionation has become an accepted and increasingly adopted dose schedule at ours and many other institutions. Nonetheless, in approximately $10-25 \%$ of cases, strict rectal dose-constraints are not met, generating a concern for increased risk of late toxicities, particularly in the context of higher dose per fraction. Furthermore, with increasing number of stereotactic ablative body radiotherapy (SABR or SBRT) schemes that employ more extreme forms of hypofractionation being investigated, there may be greater concern with regard to treatment toxicity. In these scenarios the possibility of rectal sparing with a single, mildly invasive procedure may be appealing to maximize the therapeutic index.

Although cost-effectiveness analyses in other jurisdictions suggest routine use of SpaceOAR in every patient, ${ }^{10}$ this would be challenging to justify in the current Canadian environment. Moreover, likely clinically unjustified considering only $6 \%$ of the control arm experienced grade 2 or greater gastrointestinal toxicity (only one case [1.3\%] of grade 3) in the SpaceOAR pivotal randomized study. ${ }^{6}$ Therefore, clinical feasibility and performance data in the Canadian setting will allow economic modeling accounting for the costs of rectal spacer, repeated planning imaging studies, shorter versus longer treatment duration, and associated toxicities over the full cycle of care.

We envisage an individualized approach for SpaceOAR use in patients undergoing hypofractionated IG-IMRT (see figure 3). In our institution, cases in which hypofractionated OAR dose volume constraints are not met, a new plan is generated with conventional 
fractionation (78Gy in 39 fractions) and treated accordingly. Tailored use of rectal spacer in these cases, may offer the opportunity of hypofractionation with its logistic advantages, a reduced rectal dose and a decrease in the associated risk of toxicity.

In summary, we report the first experience with SpaceOAR use for hypofractionated IGIMRT treated patients with prostate cancer. Determining the clinical scenarios, applied in the Canadian healthcare setting, where rectal spacers may be judiciously used to achieve the most cost-effective solution in terms of reduction of toxicity are warranted. 


\section{References}

1. Kachuri L, De P, Ellison LF, et al: Cancer incidence, mortality and survival trends in Canada, 1970-2007. Chronic Dis Inj Can 33:69-80, 2013.

2. Zumsteg ZS, Zelefsky MJ: Short-term androgen deprivation therapy for patients with intermediate-risk prostate cancer undergoing dose-escalated radiotherapy: the standard of care? Lancet Oncology 13:e259-e269, 2012.

3. Zelefsky MJ, Kollmeier M, Cox B, et al: Improved Clinical Outcomes with High-Dose Image Guided Radiotherapy Compared with Non-IGRT for the Treatment of Clinically Localized Prostate Cancer. IJROBP 84:125-9, 2012.

4. Pinkawa M: Spacer application for prostate cancer radiation therapy. Future Oncology 10:851-864, 2014.

5. Mariados N, Sylvester J, Shah D, et al: Hydrogel Spacer Prospective Multicenter Randomized Controlled Pivotal Trial: Dosimetric and Clinical Effects of Perirectal Spacer Application in Men Undergoing Prostate Image Guided Intensity Modulated Radiation Therapy. IJROBP 92:971-977, 2015.

6. Hamstra DA, Mariados N, Sylvester J, et al: ontinued Benefit to Rectal Separation for Prostate Radiation Therapy: Final Results of a Phase III Trial. IJROBP 97(5):976-985, 2017.

7. Catton CN, Lukka H, Gu CS, et al. Randomized Trial of a Hypofractionated Radiation Regimen for the Treatment of Localized Prostate Cancer. JCO [epub ahead of print], 2017.

8. Dearnaley D, Syndikus I, Mossop H, et al: Conventional versus hypofractionated highdose intensity-modulated radiotherapy for prostate cancer: 5-year outcomes of the randomised, non-inferiority, phase 3 CHHiP trial. Lancet Oncol 17(8):1047-60, 2016.

9. Aluwini S, Pos F, Schimmel E, et al: Hypofractionated versus conventionally fractionated radiotherapy for patients with prostate cancer (HYPRO): acute toxicity results from a randomised non-inferiority phase 3 trial. Lancet Oncol 16(3):274-83, 2015.

10. Hutchinson RC, Sundaram V, Folkert M, et al: Decision analysis model evaluating the cost of a temporary hydrogel rectal spacer before prostate radiation therapy to reduce the incidence of rectal complications. Urol Oncol 34(7):291.e19-26, 2016. 


\section{Figures and Tables}

Fig. 1. (A-B) Axial and sagittal MR-T2w images showing the prostate (red), rectum (green), and space OAR (magenta). A separation of $11 \mathrm{~mm}$ between the anterior rectal wall and the prostate was achieved with SpaceOAR. (C) Dose distribution on the corresponding co-registered planning-CT sagittal image depicts the ability of SpaceOAR to exclude a significant proportion of the rectal wall from the higher dose region.
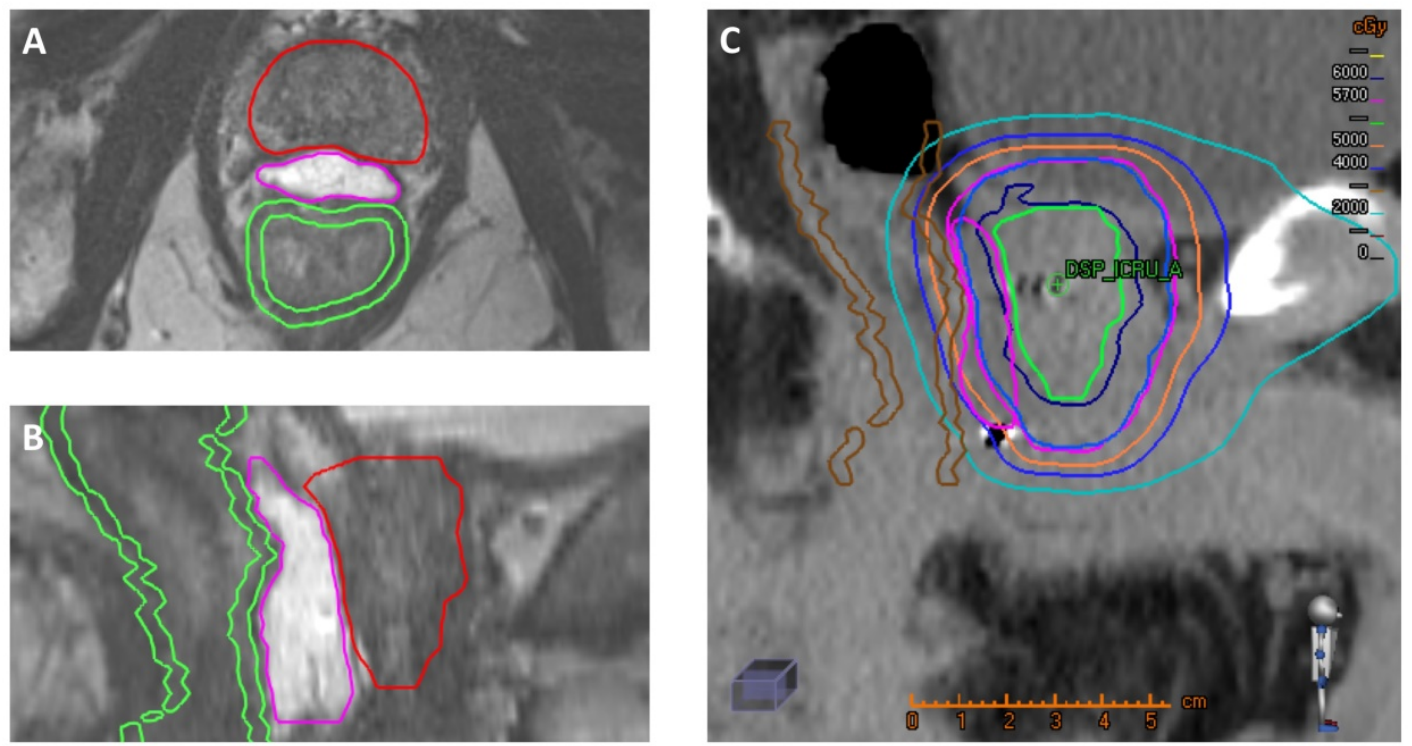
Fig. 2. Averaged DVH of prostate and rectal wall (RW) in the five patients with SpaceOAR (continuous lines), and five randomly selected historical cases treated with identical dose schedule (doted lines). Mean dose to $30 \%$ and $50 \%$ of the rectal wall significantly decreased from 45.8 and $26.9 \mathrm{~Gy}$ in the historical group compared to 27.8 and $15.3 \mathrm{~Gy}$ in the SpaceOAR patients (t-test $\mathrm{p}=0.0001$ and 0.0002 , respectively).

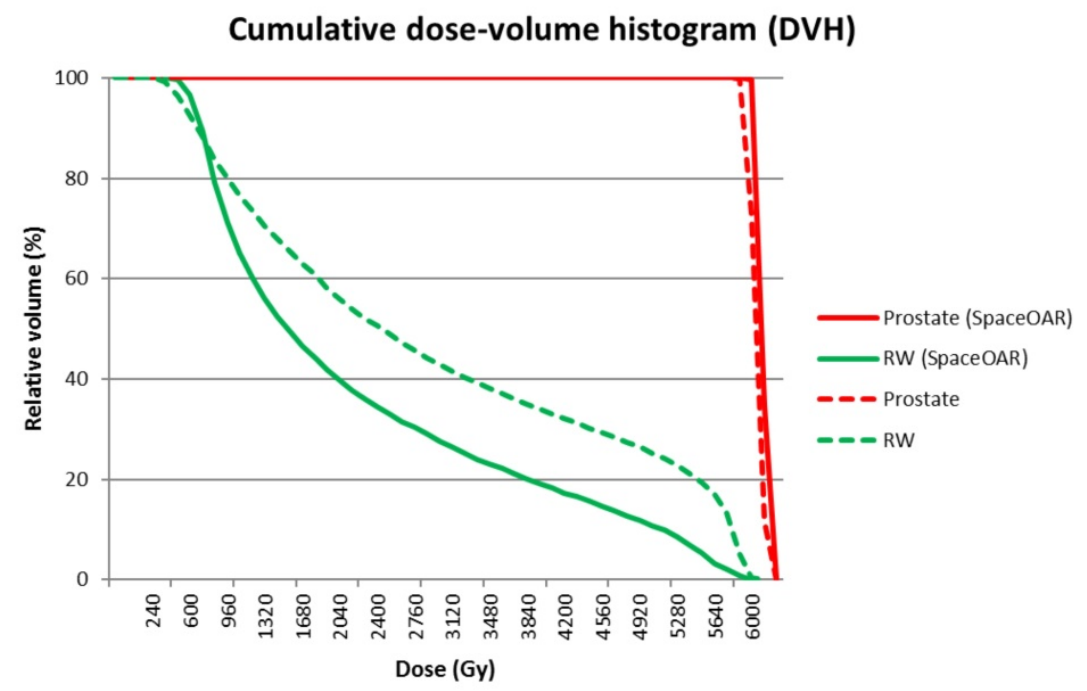


Fig. 3. Schematic representation of our proposed individualized use of SpaceOAR in patients undergoing curative-intent hypofractionated IG-IMRT for localized prostate cancer.

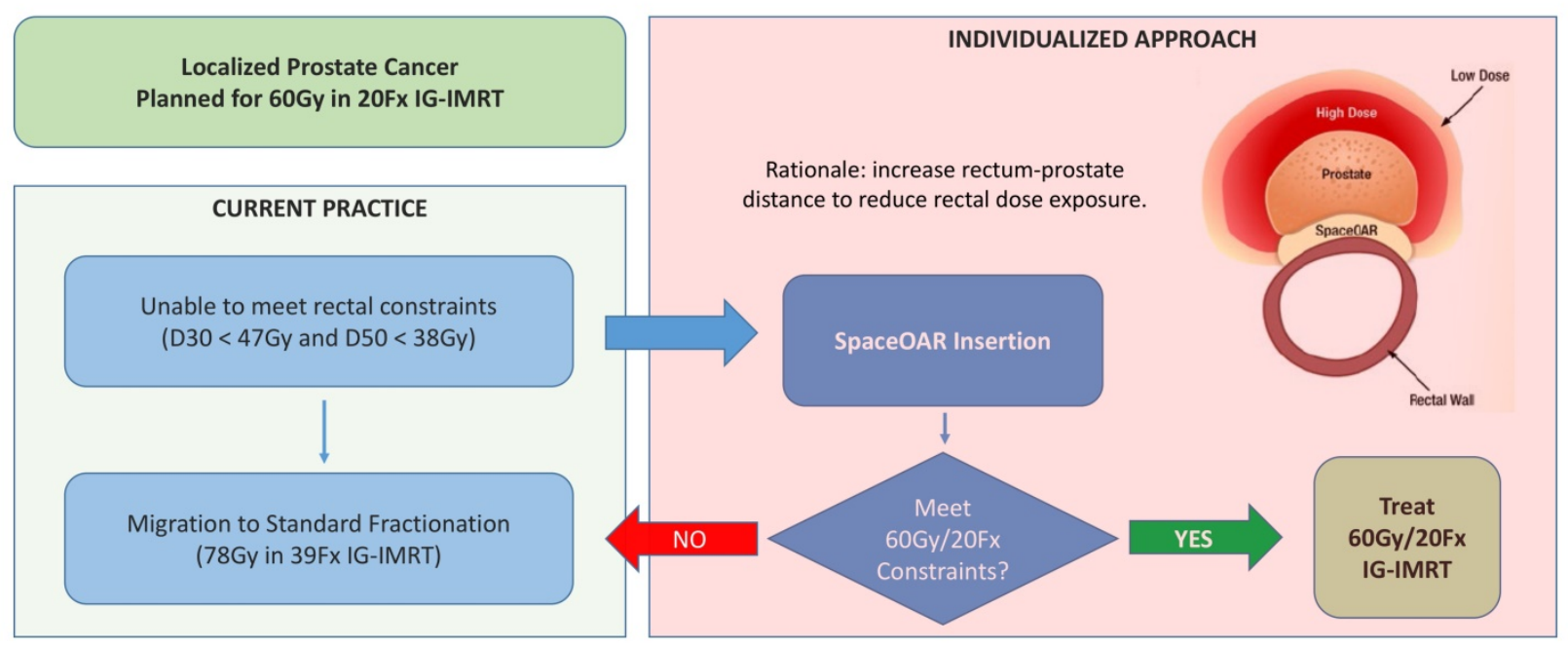

